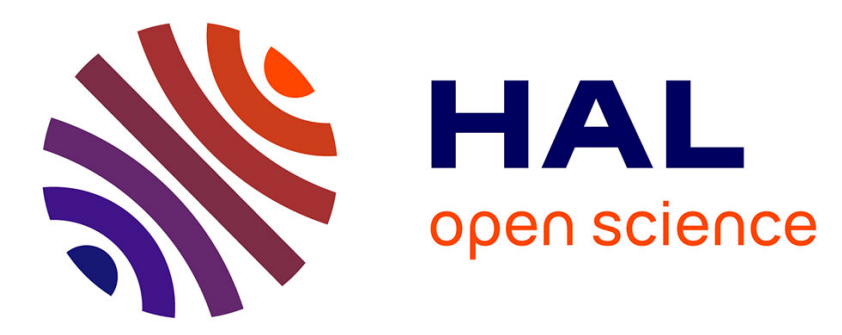

\title{
3D shape context surface registration for cortical mapping.
}

Oscar Acosta, Jurgen Fripp, Andrea Rueda, Di Xiao, Erik Bonner, Pierrick Bourgeat, Olivier Salvado

\section{- To cite this version:}

Oscar Acosta, Jurgen Fripp, Andrea Rueda, Di Xiao, Erik Bonner, et al.. 3D shape context surface registration for cortical mapping.. IEEE International Symposium on Biomedical Imaging: From Nano to Macro (ISBI'10), Apr 2010, Rotterdam, Netherlands. pp.1021 - 1024, 10.1109/ISBI.2010.5490163 . hal-00910421

\section{HAL Id: hal-00910421 \\ https://hal.science/hal-00910421}

Submitted on 28 Nov 2013

HAL is a multi-disciplinary open access archive for the deposit and dissemination of scientific research documents, whether they are published or not. The documents may come from teaching and research institutions in France or abroad, or from public or private research centers.
L'archive ouverte pluridisciplinaire HAL, est destinée au dépôt et à la diffusion de documents scientifiques de niveau recherche, publiés ou non, émanant des établissements d'enseignement et de recherche français ou étrangers, des laboratoires publics ou privés. 


\title{
3D SHAPE CONTEXT SURFACE REGISTRATION FOR CORTICAL MAPPING
}

\author{
Oscar Acosta ${ }^{1,4}$, Jurgen Fripp ${ }^{1}$, Andrea Rueda ${ }^{1,2}$, Di Xiao ${ }^{1,3}$, \\ Erik Bonner $^{1}$, Pierrick Bourgeat ${ }^{1}$, and Olivier Salvado ${ }^{1}$ \\ ${ }^{1}$ CSIRO Preventative Health National Research Flagship, ICTC, The Australian e-Health Research \\ Centre - BioMedIA, Herston, Australia \\ ${ }^{2}$ BioIngenium Research Group, Universidad Nacional de Colombia, Bogotá, Colombia \\ ${ }^{3}$ ANSTO, NSW, Australia \\ ${ }^{4}$ LTSI-INSERM U-642. Université de Rennes 1, France
}

\begin{abstract}
Deformable registration of cortical surfaces facilitates longitudinal and intergroup comparisons of cortical structure and function in the study of many neurodegenerative diseases. Non-rigid cortical matching is a challenging task due to the large variability between individuals and the complexity of the cortex. We present a new framework for computing cortical correspondences on brain surfaces based on 3D Shape Context and mean curvatures of partially flattened surfaces (PFS). Our approach is scale invariant and provides an accurate and anatomically meaningful alignment across the population. Registering PFS, instead of original cortical surfaces, simplifies the determination of shape correspondences, overcoming the problem of intersubject variability, while still guaranteeing the alignment of the main brain lobes and folding patterns. We validated the approach using 30 segmented brains from the OASIS database registered to a common space and compared the results with Freesurfer. In average, mean absolute distance of 0.36 and Hausdorff distance of 5.06 between moving and target surfaces are obtained. Further localization of labelled areas on each hemisphere demonstrated the accuracy of the technique.
\end{abstract}

Keywords: Cortical mapping, surface registration, shape context, MRI brain segmentation.

\section{INTRODUCTION}

The study of functional and morphological changes in the brain, occuring with age or during pathological processes, requires the mapping of a given population to a common space. This has proved to be a challenging task due to the convoluted geometry of the brain and the high interindividual variability [2]. Although currently the intensity-based non-rigid registration methods work well in intrasubject registration, they may not effectively address the issues arising from the variability across a population [3]. Conversely, cortical surfaces may provide a reliable representation for visualization, quantitative analysis and cortical mapping [4] as they contain anatomically meaningful features to constrain the transformations. Indeed, while there is a variability in high level foldings, gyri and sulci, the cortical shapes tend to present similar patterns at coarser levels [17]. A good matching can be consequently assured by the alignment of the lobes and major folding patterns at a coarse level.

Some of the previously proposed surface based non-rigid registration approaches use a parametric representation on a sphere, obtained either through iterative relaxation [5] or conformal mapping $[6,7]$. Further, in the spherical domain, the registration strategy relies on features such as sulcal landmarks or mean curvature $[8,9]$. In those approaches the global shape information is not explicit and

the registration is highly dependent on the selected features. An intermediate unfolded (inflated) representation of the brain provides an alternative to expose hidden sulci and to simplify the geometry for cortical mapping preserving its global shape at a coarse level. Known as partially flattened surfaces (PFS) $[10,5,11]$ they can also provide an intermediate domain to further conformal mapping [17]. Compared to the original brain surface representations, the PFS simplifies the determination of shape correspondences between subjects while avoiding a spherical mapping procedure [12].

Surface based non-rigid registration methods face several challenges including i) the choice of similarity criterion and ii) the matching and global optimization method [13]. The first one refers to the type of information extracted from the 3D surface, namely the description of local or global shape to represent the similarity. The latter challenge concerns the exploitation of the similarity information to find the best matching between the two surfaces. In a typical scheme, based on control point optimization, a further issue includes the choice of interpolation, or surface representation, in order to update the whole moving surface after control point matching. In this context, the goal of the registration is to determine the transformation such that for a finite set of control points, any control point of the moving surface is mapped onto the corresponding control point of the target surface.

Let $\mathbf{M}=\left\{\mathbf{m}_{\mathbf{i}}\right\}_{i=1 . . p}$ and $\mathbf{F}=\left\{\mathbf{f}_{\mathbf{j}}\right\}_{j=1 . . q}$ be respectively the moving and target images to be registered and $\mathbf{M}_{\mathbf{s}}=\left\{\mathbf{m}_{\mathbf{k}}\right\}_{k=1 \ldots u}$ and $\mathbf{F}_{\mathbf{s}}=\left\{\mathbf{f}_{\mathbf{l}}\right\}_{l=1 \ldots v}$ the simplified surfaces, represented in two subsets of characteristic points (control points) of $\mathbf{M}$ and $\mathbf{F}$, such that $\mathbf{M}_{\mathbf{s}} \subseteq \mathbf{M}, \mathbf{F}_{\mathbf{s}} \subseteq \mathbf{F}$. In point matching registration, after establishing bijective correspondences between the sets $\mathbf{M}_{\mathbf{s}}$ and $\mathbf{F}_{\mathbf{s}}$, exploiting a given similarity metric, the mapping is computed as a set of transformations $\mathbf{T}=\left\{\mathbf{T}_{\mathbf{k}}\right\}_{k=1 \ldots u}\left(\mathbf{T}_{\mathbf{k}} \in \mathbb{R}^{3} \rightarrow \mathbb{R}^{3}\right)$ such that $\mathbf{f}_{\mathrm{k}}=\mathbf{T}_{\mathrm{k}} \circ \mathbf{m}_{\mathrm{k}}, \forall \mathbf{m}_{\mathrm{k}}$.

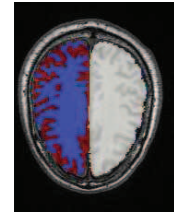

T1-W MRI

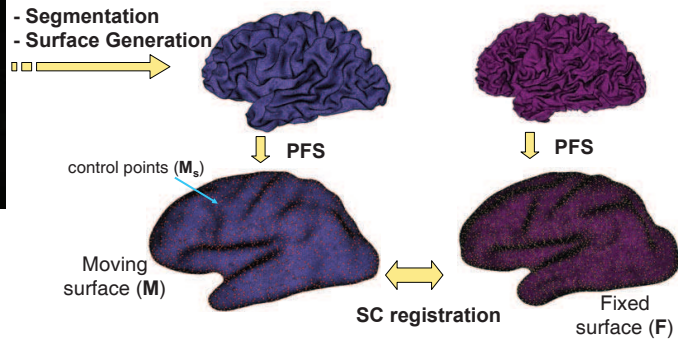

Fig. 1. Overall process for shape context cortical mapping. The moving PFS surface $\mathbf{M}$ is to be registered to the fixed PFS $\mathbf{F}$. 
In this paper we propose to exploit the highly discriminative scale-invariant properties of the shape context (SC) [14] to compute the similarity between the shapes for cortical mapping. The procedure is summarized in Fig. 1 in which after segmentation of a magnetic resonance image (MRI) a surface representing the cortex is generated. In the general pipeline, the segmentation and mesh representation are obtained with the method described in [1], however for the purpose of comparison and validation of the mapping in this paper, the segmentation and surface extraction were performed with Freesurfer [5]. In Fig. 1, this surface represents the white matter (WM) / gray matter (GM) interface, which is to be registered towards another surface or a common template. Firstly, the cortical surfaces representing the brain hemispheres are partially flattened, obtaining the moving and fixed PFS surfaces, $\mathbf{M}$ and $\mathbf{F}$, respectively. Secondly, in a two level optimization step, a bijective matching between the simplified surfaces $\mathbf{M}_{\mathbf{s}}$ and $\mathbf{F}_{\mathbf{s}}$ is computed using the shape context and the mean curvatures. Finally, a Thin Plate Spline (TPS) transformation [15] maps $\mathbf{M}$ into $\mathbf{F}$ using the set of control point transformations $\mathbf{T}$. The major contribution of the shape context lies in the global shape characterization at a local level for each single point. Combined with the curvature, our method produces an anatomically meaningful and scale-invariant matching between the lobes and the major folding patterns. In the remainder of the paper we explain first how we obtain the PFS (Section 2.1), followed by a description of the shape context (Section 2.2) and the optimization scheme (Section 2.3). Validation experiments, including comparison with Freesurfer, are performed on 30 segmented brains (left and right hemispheres).

\section{METHOD}

\subsection{Partially flattened surfaces (PFS)}

A number of methods have been proposed in the literature for unfolding or flattening cortical surfaces $[10,5,11]$. In order for a flattening to be useful, it must preserve local and global metrics such as triangle angles and areas. We previously performed a thorough comparison of different methods in [16]. For our purposes we selected the method based upon CARET [10], already used in [17], which has been proved to preserve local and global geometric metrics.

Thus, the cortical surface is iteratively deformed at each vertex according to $\mathbf{m}_{i}^{t+1}=(1-\lambda) \mathbf{m}_{i}^{t}+\lambda \overline{\mathbf{m}}_{i}^{t}$, where $\mathbf{m}_{i}^{t}$ is the position of vertex $i$ for iteration $t, \lambda$ is a scalar in the range $[0,1]$ and $\overline{\mathbf{m}}_{i}{ }^{t}$ represents the average vertex position of $\mathbf{m}_{\mathbf{i}}{ }^{t}$, given by

$$
\overline{\mathbf{m}}_{i}^{t}=\frac{1}{\sum_{j \in N_{i}} \mathbf{S}_{j}^{t}} \sum_{j \in N_{i}} \mathbf{S}_{j}^{t} \mathbf{c}_{j}^{t}
$$

where $N_{i}$ is the set of all triangles containing $\mathbf{m}_{i}, \mathbf{c}_{j}$ is the center of triangle $j$ and $\mathbf{S}_{j}$ is its area. This process moves all mesh vertices towards the weighted average of the centers of their surrounding triangles. The deformation progresses until the global mean curvature $\bar{k}$ drops below a predefined threshold (typically $\bar{k}=0.13$ ), obtaining similar shapes between moving and fixed surfaces[17]. The sulcal depth maps or the curvature at an intermediate level can be used as local landmarks.

Fig. 2 illustrates the inflation results at different iterations with the sulcal depth mapped into the final surface. Fig.3 shows the different steps in the evolution of the PFS and the final curvature map. In this paper the local mean curvature is computed and stored as a feature at each point of the surface. Finally, the deformation continues until the surfaces $\mathbf{M}$ and $\mathbf{F}$ reach a given similar level of $\bar{k}$.

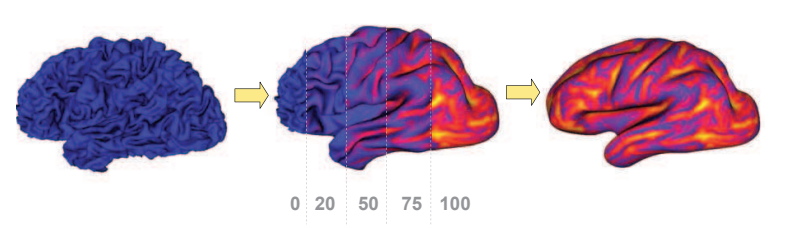

Fig. 2. Original cortical mesh is iteratively flattened to a PFS. From left to right $0,20,50,75$ and 100 iterations. The sulcal depth at each point is represented in the final mesh.

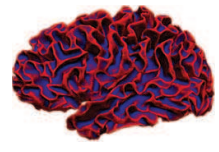

(a)

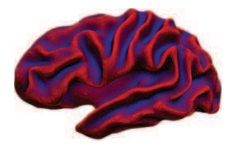

(b)

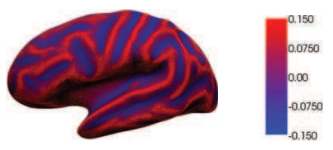

(c)
Fig. 3. Original cortical mesh (a) is flattened to a PFC. Mean curvatures are calculated at an intermediate level of inflation (b) and mapped onto the final PFC (c).

\subsection{Shape context}

This step aims to find the corresponding points between the moving and fixed PFS. The shape context is a shape descriptor that, for a single surface, captures the distribution of points over relative positions of the global shape points. This characterization is invariant to scale and rigid transformations, naturally leading to a highly discriminative and robust score for measuring shape similarities. The shape context was first introduced in [14] within the 2D pattern recognition field, and aimed to match point clouds representing similar patterns. Further modifications to the method appeared in [19] and $3 \mathrm{D}$ matching of features have been extended to work with thoracic images [20].

In the proposed method, after PFS, two simplified surfaces $\mathbf{M}_{\mathbf{s}}$ and $\mathbf{F}_{\mathbf{s}}$ are obtained using an algorithm which iteratively contracts vertex pairs while minimizing geometric errors [21]. They constitute the control points of the moving and fixed surfaces and for which the shape context is computed. Thus, for a given point $\left\{\mathbf{m}_{\mathbf{k}}\right\}_{k=1 . . u} \in$ $\mathbf{M}_{\mathbf{s}}$, its shape context is the $3 \mathrm{D}$ histogram of the relative $3 \mathrm{D}$ polar coordinates $(\vec{r}, \theta, \phi)$ of the remaining $u-1$ points. As in [18], in order to be more sensitive to nearby points, we use a log-polar coordinate system. In our case we build a 3D histogram with $R_{\vec{r}}$ equally spaced log-radius bins and $u_{\theta}$ and $v_{\phi}$ equally spaced angle bins. Since the intracranial volume may considerably differ across a population, an additional normalization of the shape context is performed to obtain scale invariance by normalizing all the radial distances $\operatorname{dist}\left(\mathbf{m}_{\mathbf{k}}, \mathbf{m}_{\mathbf{l}}\right)_{k, l=1 . . u},\{k \neq l\}$ by the mean distance between all the point pairs in the shape: $\frac{1}{u} \sum_{k, l} \operatorname{dist}\left(\mathbf{m}_{\mathbf{k}}, \mathbf{m}_{\mathbf{l}}\right)_{k, l=1 . . u},\{k \neq l\}$.

\subsection{Cost function and matching}

The cost $C_{i, j}$ of matching the point $\mathbf{m}_{\mathbf{i}}$ from the simplified surface $\mathbf{M}_{\mathbf{s}}$ with the point $\mathbf{f}_{\mathbf{j}}$ from $\mathbf{F}_{\mathbf{s}}$ is given by the $\chi^{2}$ statistics as

$$
C_{i, j}=\frac{1}{2} \sum_{k=1}^{K} \frac{\left[h_{i}(k)-g_{j}(k)\right]^{2}}{h_{i}(k)+g_{j}(k)}
$$

where $h_{i}(k)$ and $g_{j}(k)$ are the histograms (shape context) at the points $i$ and $j$ of the moving $\mathbf{M}_{\mathbf{s}}$ and fixed $\mathbf{F}_{\mathbf{s}}$ shapes, respectively, 


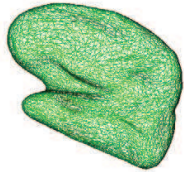

(a) $\mathrm{M}_{\mathrm{s}}$

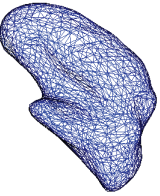

(b) $\mathbf{F}_{\mathrm{s}}$

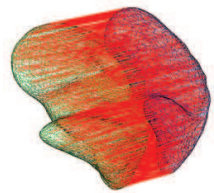

(c) Control point matching
Fig. 4. Example of control point (CP) matching using shape context. Target $\mathbf{F}_{\mathbf{s}}$ and moving $\mathbf{M}_{\mathbf{s}}$ simplified surfaces and their computed correspondences.
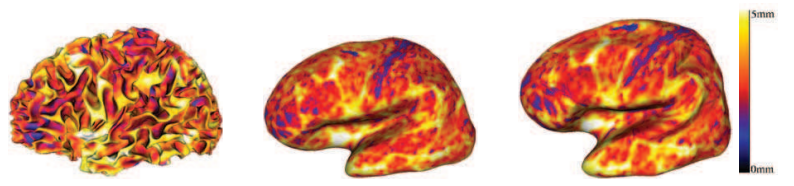

Fig. 5. Example of propagated cortical thickness values from one individual to a common template after registration of PFS.

and $K$ is the total number of bins. Given the individual costs $C_{k, l} \in$ $[0, . ., 1]$ between all pairs of points, the next step is to find the perfect matching by minimizing the total cost of the bijective correspondences $\mathbb{H}=\sum C$. This is done within a one to one point matching step with the Hungarian algorithm [22]. Fig. 4 shows the result of matching for two point sets $\mathbf{M}_{\mathbf{s}}$ and $\mathbf{F}_{\mathbf{s}}$. After the correspondences are found, the set of transformations $\mathbf{T}=\left\{\mathbf{T}_{\mathbf{k}}\right\}_{k=1 . . u}$ can be easily computed. Finally, a transformation using a thin plate spline model (TPS)[15] calculated from the control points, achieves the interpolation of the corresponding moving shape $\mathbf{M}$ onto $\mathbf{F}$. This yields a surface globally aligned by lobes and locally following the major folding patterns.

After the first matching step, the surfaces are finely realigned by recomputing a new cost function for the points $\mathbf{m}_{\mathbf{i}}$ and $\mathbf{f}_{\mathbf{j}}$

$$
\mathbb{C}=\alpha_{1} C_{i, j}+\alpha_{2}\left\|\kappa_{i}-\kappa_{j}\right\|
$$

which includes the local landmarks (mean curvatures,..) $\kappa_{i}$ and $\kappa_{j}$ and where $\alpha_{1}$ and $\alpha_{2}$ weight the contribution of each term. The resultant matching minimizes $\mathbb{H}=\sum_{i} \mathbb{C}$. The registration allows then the interindividual propagation of information such as cortical thickness for statistical analysis. Fig. 5 shows an example of cortical thickness information from a single individual transferred to a common template.

\section{EXPERIMENTS AND RESULTS}

From the OASIS database [23], we randomly selected 30 young healthy individuals. The magnetic resonance (MR) scans were T1w magnetization prepared rapid acquisition gradient echo (MPRAGE) with isotropic $1 \mathrm{~mm}^{3}$ resolution. In this paper the tissue segmentation and the (WM/GM) surfaces were computed using FreeSurfer. Thus, for each individual we obtained separated surfacical representations for left and right hemispheres. The overall method was used to register the surfaces to a common template, which was obtained after segmentation of the BrainWeb atlas[24] with FreeSurfer.

In the first experiment, we investigated both the effect of the number of control points employed to represent the simplified surfaces and the influence of the curvature on the registration results. Mean absolute (MAD) and Hausdorff (HD) distances [25] between moving and target surfaces were used to assess the accuracy of our method to register two PFSs. Table 1 presents a summary of these results. On average, between 500 and 1000 points the accuracy was improved by $33 \%(p<0.0001)$ and by $32 \%(p<0.0001)$ between 1000 and 2000 points for the left hemisphere and $23 \%(p<0.0001)$ and $27 \%(p<0.01)$ respectively for the right hemisphere. As expected, with less simplified surfaces the alignment is better. A tradeoff between computational requirements and accuracy exists which depends on the considered application. When the curvature step is included, the improvement is statistically significant in terms of MAD. In our experiments it was $24 \%$ and $49 \% p<0.001$ for left and right hemispheres. The area coincidence between the moving and fixed images after registration reached $99 \%$.

In the second experiment, we compared the localization of Freesurfer labeled regions (obtained as described in [26]) after registration. The Jaccard's coefficient measured as the relation between the intersection and union of areas of labeled regions was used as similarity metric. Paired t-tests per region were used to test for statistical significance. Overall, our method is comparable with the registration provided with Freesurfer. In 6 regions of the left hemisphere and 6 of the right hemisphere the similarity metric was higher than Freesurfer. In other regions the achieved accuracy was lower than with Freesurfer but in most of them these differences were not statistically significant, excepted the entorhinal cortex, middle temporal, pastriangularis and transverse temporal [26] in both hemispheres.

In the third experiment, we evaluated the accuracy of the proposed method to match the major folding patterns (gyri, sulci) across the patients (Fig. 6). Thus, using the mean curvature maps (Fig. 3), we automatically labelled each individual's intermediate inflated surface in two classes: i) The major sulcal $(\mathbb{S})$ and ii) the gyral $(\mathbb{G})$ patterns, from the sum of negative and positive mean curvatures, respectively. Those maps were obtained during the flattening process as a threshold of $\Im(t)=\sum_{i=1}^{t} \kappa_{i}^{t}$, where $t$ is the number of iterations and $\kappa_{i}^{t}$ is the mean curvature of the point $i$. Thus, $\mathbb{S}=1$, if $\Im(t)>0$ and $\mathbb{G}=1$, if $\Im(t)<=0$. The coincidence of the folding patterns indicates the quality of the matching after the registration of the 30 individuals to the common template. $62.30 \%$ of the points in the left hemisphere were classified as belonging to the same folding pattern (gyrus or sulcus) in at least 20 cases (66\% of the individuals). $63.06 \%$ in the right hemisphere. Fig. 6 illustrates the resultant labeling probability maps after registering the whole population to the same template.

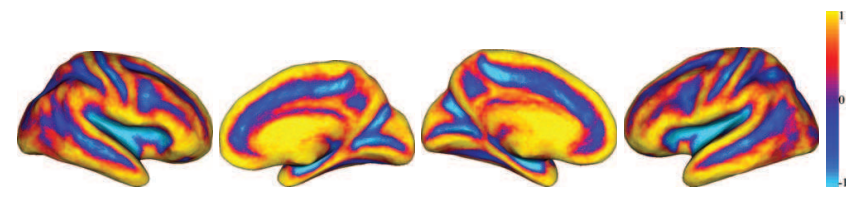

Fig. 6. Folding patterns probability maps generated after registration of all the individuals to the same template. Left and right hemispheres, lateral and medial views. Major gyral (hot colors) and sulcal patterns (cold colors).

These results suggest that the proposed shape context-based non-rigid registration scheme achieves accurate and reliable registration to be used in cortical mapping. The method is comparable with Freesurfer in terms of accuracy in aligning most of the prelabeled Freesurfer regions. Overall, our method and FreeSurfer performed better in the left hemisphere. When curvature is used the method significantly improves the accuracy. A multiscale approach is the next step in the current implementations in which iteratively the folds are matched starting at a coarse level and going back until the original resolution. 
Table 1. Averaged Hausdorff distance (HD), Mean Absolute Distance (MAD) and \%Area coincidence after registration between moving M and fixed $\mathbf{F}$ surfaces. Mean(standard deviation) of 30 left and 30 right hemispheres, as the number of sampling points vary and when the landmark curvature $(\mathrm{w} / \kappa)$ is included.

\begin{tabular}{l|c|c|c|c|c|c|c|c}
\hline \multirow{2}{*}{ NPts } & \multicolumn{5}{|c|}{ Left h } & \multicolumn{4}{c}{ Right h } \\
\cline { 2 - 9 } & 500 & 1000 & 2000 & $\mathrm{w} / \kappa$ & 500 & 1000 & 2000 & $\mathrm{w} / \kappa$ \\
\hline \hline HD & $8.45(1.9)$ & $7.25(1.97)$ & $6.29(1.71)$ & $5.06(1.17)$ & $14.97(7.13)$ & $13.24(7.83)$ & $13.11(8.25)$ & $5.31(1.34)$ \\
MAD & $0.74(0.05)$ & $0.49(0.04)$ & $0.35(0.03)$ & $0.36(0.03)$ & $1.17(0.58)$ & $0.86(0.61)$ & $0.74(0.83)$ & $0.37(0.02)$ \\
\%A & $98.08 \%$ & $98.45 \%$ & $98.72 \%$ & $99.38 \%$ & $97.02 \%$ & $97.72 \%$ & $98.8 \%$ & $99.6 \%$ \\
\hline
\end{tabular}

\section{CONCLUSION}

In this paper we have proposed an approach for non-rigid registration of cortical surfaces based on both the shape context and the mean curvature. Shape context yields a discriminative score of shape that incorporates global shape information into a local descriptor. Combined with curvature, it achieves an anatomically meaningful matching between the lobes and the major sulcal lines. Any other landmark representing foldings such as sulcal depth can be used. This scheme uses PFS representations whose shape is similar across different brains. As comparison with FreeSurfer has shown that the labeling of major folding patterns is preserved, our method represents an alternative for cortical mapping across a population. Future work also includes the extension to multifeature support in which not only anatomically signatures are matched, but also similar functional patterns. We also intend to perform longitudinal clinical studies on neurological disorders including Alzheimer's disease. We are particularly interested in comparing cortical thickness between a population of healthy individuals with an Alzheimer's disease cohort. After mapping of the cortical thickness maps to a template, statistical analysis per vertices can be performed.

\section{REFERENCES}

[1] Acosta, O.,et al: Automated voxel-based 3D cortical thickness measurement in a combined Lagrangian-Eulerian PDE approach using partial volume maps. MedIA 13 (2009) 730-743

[2] Mangin, J.F., et al: A framework to study the cortical folding patterns. NeuroImage 23(Supplement 1) (2004) S129 - S138 Mathematics in Brain Imaging.

[3] Hellier, P. et al: Retrospective evaluation of intersubject brain registration. IEEE TMI 22(9) (2003) 1120-1130

[4] Tosun, D. et al: Cortical surface segmentation and mapping. NeuroImage 23(Supplement 1) (2004) S108 - S118 Mathematics in Brain Imaging.

[5] Fischl, B. et al: Cortical Surface-Based Analysis II: Inflation, Flattening, and a Surface-Based Coordinate System. NeuroImage (1999) 195-207

[6] Angenent, S. et al: On the Laplace-Beltrami operator and brain surface flattening. IEEE TMI 18 (1999) 700-71

[7] Gu, X., et al: Genus zero surface conformal mapping and its application to brain surface mapping. IEEE TMI 23(8) (2004) 949-958

[8] Yeo, B.T. et al: Spherical demons: Fast surface registration. In: MICCAI 2008. Vol. 5241., Springer (2008) 745-753

[9] Zou, G. et al: Non-rigid surface registration using spherical thin-plate splines. In: MICCAI 2007. Vol. 4791., Springer (2007) 367-374
[10] Drury, H. et al: Computerized mappings of the cerebral cortex: A multiresolution flattening method and a surface-based coordinate system. J Cognit Neurosci 8(1) (1996) 1-28

[11] Pons, J.P. et al: Area preserving cortex unfolding. In: MICCAI 2004. Vol. 3216., Springer (2004) 376-383

[12] Eckstein, I. et al: Generalized surface flows for deformable registration and cortical matching. In: MICCAI 2007 692-700

[13] Audette, M.A. et al: An algorithmic overview of surface registration techniques for medical imaging. Medical Image Analysis 4(3) (2000) 201-217

[14] Belongie, S., Malik, J.: Matching with shape contexts. Content-based Access of Image and Video Libraries, 2000. Proceedings. IEEE Workshop on (2000) 20-26

[15] Bookstein, F.L.: Principal warps: thin-plate splines and the decomposition of deformations. IEEE TPAMI 11(6) (1989) 567585

[16] Bonner, E. et al: "A quantitative comparison of three methods for inflating cortical meshes," in 2009 IEEE International Symposium on Biomedical Imaging: From Nano to Macro, Boston, Massachusetts, USA, June, pp. 1338-1341.

[17] Tosun, D. et al: Mapping techniques for aligning sulci across multiple brains. MedIA (2004) 295-309

[18] Belongie, S. et al: Shape matching and object recognition using shape contexts. IEEE TPAMI 24 (2002) 509-522

[19] Mori, G. et al: Efficient shape matching using shape contexts. IEEE TPAMI 27(11) (2005) 1832-1837

[20] Urschler, M. et al: Registering 3D lung surfaces using the shape context approach. Proc. Medical Image Understanding and Analysis (2004) 212-215

[21] Garland, M. et al: Surface simplification using quadric error metrics. In: SIGGRAPH '97, New York, NY, USA, ACM Press/Addison-Wesley Publishing Co. (1997) 209-216

[22] Kuhn, H.W.: The hungarian method for the assignment problem. Naval Research Logistics Quarterly (2) (1955) 83-87

[23] Marcus, D.S. et al: Open access series of imaging studies (OASIS): Cross-sectional MRI data in young, middle aged, nondemented, and demented older adults. J Cogn Neurosci 19 (2007) 1498-1507

[24] Collins, D. et al: Design and construction of a realistic digital brain phantom. IEEE TMI 17(3) (1998) 463-468

[25] Gerig, G. et al: Valmet: A new validation tool for assessing and improving 3D object segmentation. In: MICCAI 2001, Springer (2001) 516-523

[26] Desikan, R.S. et al: An automated labeling system for subdividing the human cerebral cortex on MRI scans into gyral based regions of interest. NeuroImage 31(3) (2006) 968-980 